\title{
The International Dimension of Climate Change Policy
}

\author{
Michael Finus • Christos Kotsogiannis • \\ Steve McCorriston
}

Published online: 4 October 2013

C Springer Science+Business Media Dordrecht 2013

Climate change is a major challenge to international cooperation, as emphasized for instance by the Stern Review and various IPCC reports (Stern 2006; IPCC 2007) but also many others. One of the main problems of achieving cooperation under international climate agreements is free-riding. Countries have an incentive to adopt a non-cooperative behaviour. Emission reduction constitutes a public good. No country can be excluded to benefit from the emission reduction of other countries. Moreover, by not contributing to emission reduction, a country saves on abatement cost. The Kyoto Protocol ran out in 2012, but all efforts to negotiate an effective follow-up protocol have failed up to now. The reason is not that no negotiations would have taken place, but simply that no proposal was yet formally adopted which sets emission ceilings such that greenhouse gas emissions are stopped from further growing. What is required are substantial cut backs on emissions in order to meet the widely accept target of not allowing the average global temperature to increase beyond $2{ }^{\circ} \mathrm{C}$.

The seven papers in this special issue look at various important aspects related to the international dimension of climate policy. The first five papers are theoretical papers, the last two papers employ a climate simulation model combined with a game theoretic module to derive

The papers that appear in this special issue were all contributed papers to the "Environment and Sustainability Forum" that took place at the University of Exeter in April 2011. All papers have been subjected to the standard Environmental and Resource Economics refereeing process. We thank the participants of the conference and the Editor of ERE, Ian Bateman, for his encouragement and advice. We also thank the University of Exeter Business School for its generous support and, certainly not least, the referees who provided invaluable help in the evaluation process of these papers. Finally, the guest editors would like to thank Mr Alaa Khourdajie for his research assistance.

\footnotetext{
M. Finus ( $\square)$

Department of Economics, University of Bath, 3 East, Bath BA2 7AY, UK

e-mail: m.finus@bath.ac.uk

C. Kotsogiannis $\cdot$ S. McCorriston

Department of Economics, University of Exeter, Streatham Court, Rennes Drive,

Exeter EX4 4PU, UK

C. Kotsogiannis

CESIfo, Munich, Germany
} 
their results. Issues that are covered are for, instance, the problem of long-term commitment of governments to a proactive climate policy, the role of R\&D in clean technologies, in terms of how they are induced through various policy instruments but also how spillovers and adoption of green technology can support international cooperation, the role of ancillary benefits for the success of climate agreements, the relationship between trade and climate policy, the design of supply-side climate policies to address the green paradox, the driving forces that support or undermine a unilateral climate policy without full participation and finally the role of fat tail distributions of uncertain damages on the performance of partially cooperative climate agreements. In the following, we summarize these papers briefly and encourage the reader to have a careful read of this excellent selection of papers, which span a wide range of important topics in the current debate about an effective way forward in addressing the climate change problem.

\section{Ulph, A. and D. Ulph, Optimal Climate Change Policies When Governments Cannot Commit}

Addressing climate change raises a wide range of issues which are intrinsically uncertain, the nature of this uncertainty exacerbated by the long-term nature of the climate change problem. Given that there are many dimensions to climate change uncertainty, questions emerge as to whether a "wait and see" approach is preferable to action now. For example, since we are unsure of future climate change projections and new technology that may be available, there may be a higher pay-off to delaying investment and developing policies to address these issues. An alternative view is that the "deep" uncertainty about climate change is no excuse for (at least some) action now. The paper by Ulph and Ulph presents an argument where inter-temporal uncertainty should lead to policy-makers creating greater incentives in the short-term with the result that commitment to addressing climate change is 'front loaded'. The specific aspect of uncertainty in their paper is associated with the political process: given that governments are in power for a limited period of time, it may be the case that future governments may not have the same commitment to addressing environmental issues relative to the current one. The uncertainty here may reflect a wide range of factors that may affect a government's priorities (for example, recession, financial crisis and so on) such that the emphasis on addressing climate change issues becomes diluted. While the current government may be clear about their environmental priorities and set environmental taxes consistent with this, the uncertainty associated with future governments' intentions causes responses to differ compared with the case where this uncertainty about political priorities would not exist.

Ulph and Ulph set up a two period model where the government in the current period has a bias towards environmental issues; there is, however, uncertainty about whether the bias will be sustained in the second period. The current government sets an environmental tax, but there is uncertainty what this tax will be in the next period i.e. there is a lack of commitment because the current government may not be in power in the next period. On the real side of the economy, there is a single firm that invests in $\mathrm{R} \& \mathrm{D}$ (the single firm assumption being employed to limit the externality associated with the firm not capturing the monopoly rent associated with invention i.e. the $R \& D$ externality does not exist). In principle, the environmental tax should encourage the firm to invest in more $R \& D$, which will help mitigate the climate issue in the second period. But the uncertainty held by the firm about the priorities of the (different) government in the second period causes the firm to under-invest such that the political uncertainty 
is associated with too little $\mathrm{R} \& \mathrm{D}$, irrespective of the optimal policy levels chosen by the current government.

In this context, the policy solution is to subsidise $R \& D$; note that the $R \& D$ subsidy, in the presence of the political uncertainty, countervails the potential change in bias of the next government. It is necessary because the private firm is wary of the changing priorities of the government while the current government wants to ensure that there is some commitment to addressing environmental issues. Unlike a 'wait and see' approach, the R\&D subsidy serves to embed the R\&D now in face of the potential lack of commitment over environmental policy.

Ulph and Ulph deal with a very real challenge: governments are typically short-lived (at least in the context of addressing climate change issues) and the reality of policy-making is that climate change has to compete with many other (often more immediate) issues on the government's agenda. This is not to interpret the issue as the government (or the public that voted for it) caring less but that the policy agenda can be influenced by unforeseen events. This is different from the 'wait and see' scenario as the political uncertainty arises from the lack of commitment in an inter-temporal context where the government may change and the bias towards environmental issues may change with it.

In the Ulph and Ulph set up, the solution to this lack of commitment is a subsidy on R\&D, but the issue raises a more general question: if the issue is fundamentally one of political commitment, what alternatives are available that could effectively "tie the hands" of policymakers whatever the issues are faced by this or the subsequent governments? One possibility is a regulator that is independent of government (and therefore safeguarded from the short-lived constraints governments often face); another possibility is the commitment to international environmental agreements where any commitment made would be binding. These options are, of course, not problem-free but the question posed by the Ulph and Ulph paper highlights an important question about sustaining commitment to environmental policies over time.

The authors suggested a number of possible extensions. One obvious extension would be to model a stock-externality problem with emissions also in period one. Another one would be to study a multi-country problem and consider whether joining the IEA gives a form of future commitment. One could also take into account the uncertainty about climate change and how the possibility of getting better information in the future may affect current policies.

\section{Endres, A. and B. Rundshagen, Incentives to Diffuse Advanced Abatement Technology Under the Formation of International Environmental Agreements}

New technology and the incentives associated in delivering it, features prominently in the paper by Endres and Rundshagen. In their context, there are two aspects to new technology; first, the differences in abatement technology that exist across countries; second, given that these differences exist, the issue of whether to diffuse and adopt better technology also becomes important. Focussing on diffusion and adoption, alternative environmental instruments will impact differently on the decisions of firms which, in turn, will impact on the effectiveness of these policies. Endres and Rundshagen address these issues in the context of international negotiations and, in doing so, also provide another insight into the (non-) equivalence between environmental taxes and emission quota schemes.

To see the central notion in the Endres and Rundshagen paper, consider two firms in a national setting where one firm (with the superior technology) decides on diffusion, and the other decides on adoption. Suppose governments negotiate an environmental tax with the firms knowing that if they diffuse and adopt, the tax rate can fall. In this context, 
there are clear incentives to diffuse and adopt and, commensurate with this, socially optimal tax rates are chosen. Emission quotas, however, do not lead to the same outcome: if an emission limit is set by the government, the spread of new technology could lead to a stricter emission target (a higher tax equivalent); since the new technology means that stricter targets can be more easily met, this impacts negatively on the willingness of firms to diffuse and adopt. It is this inter-play between policy instruments and the incentives to diffuse and adopt that is the central focus of the paper by Endres and Rundshagen. Their specific contribution is to take these issues to the setting of international environmental negotiations where the issues become more complex and where decisions relating to diffusion and adoption not only involves technology transferring across borders but also the level of taxes and emission targets in the negotiations of an international environmental agreement.

Specifically, in the international setting, there are two countries where one firm exists in each but where one of these firms has the superior technology and where the incentives to diffuse and adopt now involve cross-border issues. In the context of international negotiations, one country is labelled as the 'bottleneck' country, where this is defined as the country that is willing to make the lowest offer either in terms of a uniform emission tax or uniform emission reduction quota. Under the environmental tax regime, the 'bottleneck country' is the country with the superior technology; the main outcome is low taxes (or at least taxes below the social optimum) and low diffusion since the burden of higher taxes would fall mainly on the superior technology country which in effect is subsidising the other country. This leads to low taxes and lower diffusion to limit this cross-country subsidisation effect. This contrasts with the national setting since there are always incentives to diffuse and adopt as it would lead to lower taxes in subsequent periods. Under the emission quota regime, the 'bottleneck country' is the country with the inferior technology which would negotiate for less strict emission levels. Incentives to adopt are now weaker since adoption of the new technology will result in tighter emission levels being set in the future.

Comparing the negotiation outcome of the two regimes in the case of asymmetric damage functions, the equilibrium in terms of pollution abatement as well as technology diffusion and adoption may be welfare superior in the quota regime compared to the tax regime, despite the quota regime's cost-ineffectiveness. This may hold in the case of identical damage functions. The reason is that the negotiated equilibrium emission reduction might be higher in the case of countries negotiating quotas instead of taxes.

Setting the specific features of the model aside, Endres and Rundshagen address two key issues that are important for future research. First, R\&D issues are not just about the development of new technology but also about diffusion and adoption and even where new technology exists, we also have to think about the incentives associated with these decisions. There is also a potentially important inter-play between these decisions and the choice of policy instruments. Second, the insights produced here highlight another dimension which is of relevance to research on international environmental agreements. Specifically, the issues are not confined about whether to join an international agreement and what policy instruments and targets to set but also about the diffusion and adoption of technology across borders and how the incentives associated with the latter can, in themselves, impact on the choice of instruments and the policy levels to set.

The authors suggest that future research may look at the effect of combinations of policy instruments such as using a subsidy instrument to encourage diffusion and adoption of new technology. Further extensions may consider other forms of how technical progress emerges and property rights issues associated with new technologies. 


\section{Finus, M. and D. T. G. Rübbelke, Public Good Provision and Ancillary Benefits: The Case of Climate Agreements}

Combating global warming will not only generate global public benefits (primary benefits) of slowing climate change, but also private benefits (secondary benefits), sometimes referred to as ancillary benefits or co-benefits. Ancillary benefits stem from mitigation measures which, as a side-effect, also reduce for instance local pollution. Empirical evidence suggests that the secondary benefits can be quite large, in particular in developing countries, and may even exceed the primary benefits. Intuition would suggest that if governments take these secondary benefits into consideration, this should increase their willingness to participate in a climate agreement. In other words, making governments aware of ancillary benefits of climate policy should be conducive to the success of climate agreements. Whether this intuitive reasoning is correct is the central question analysed in Finus and Rübbelke in this issue. The authors use a simple two-stage non-cooperative coalition formation model where in the first stage countries decide upon membership in an agreement and in the second stage choose their abatement levels. The public good part of the benefits from mitigation calls for policy coordination and hence makes free-riding attractive as in the standard model.

The authors show that the more important ancillary benefits are in governments' objective function, the higher will be global abatement. This is true in the non-cooperative Nash equilibrium and the social optimum, but is also true for partial cooperation where some countries cooperate but maybe not all. However, the question is whether ancillary benefits make a difference in relative terms. Therefore, the authors measure global welfare (the sum of payoffs over all players) in relative terms which is called the closing the gap index. This index measures the difference between what is achieved in a self-enforcing climate agreement and the non-cooperative equilibrium, in relation to the gap between the social optimum and the non-cooperative equilibrium. As analytical solutions for general payoff functions cannot be obtained, the authors consider three model versions, which have been frequently analysed in the literature. The first version assumes linear benefits and linear costs from abatement, the second version linear benefits and quadratic costs and the third version quadratic benefits and quadratic costs. Benefits comprise primary benefits from global abatement and secondary benefits from own abatement.

The authors show that participation decreases in version 1, remains constant in version 2 and may slightly increase at the margin in model version 3 . In terms of relative welfare, the closing the gap index decreases in model version 1 , remains constant in version 2 and may increase or decrease in model version 3, though any increase will only be marginal. In the first model, ancillary benefits lead to a smaller coalition as they reduce the threshold of countries necessary for an agreement to be profitable. In the other two models, ancillary benefits increase not only abatement and payoffs of coalition members but also of outsiders, which leaves the free-rider incentives (more or less) unchanged. The rationale is that countries taking the private ancillary benefits to a greater extent into account will undertake more emission reduction, irrespective of an international agreement. In other words, these benefits may provide an additional incentive to participate in international agreement but also an additional incentive to free-ride. In this respect, the counter-intuitive result appears intuitive, and in line with previous papers which showed that if agreements have to be self-enforcing, though they improve upon the non-cooperative outcome, fall short of the globally optimal solution. Hence, unfortunately, making governments aware of the importance of ancillary benefits of climate policy will not do the trick to unlock the deadlock of current climate change negotiations; this will require other measures. 


\section{Vlassis, N., The Welfare Consequences of Pollution-Tax Harmonization}

A key concern of countries contemplating a carbon pricing policies is the fear that their competitive position will be jeopardised by 'carbon leakage' as production might shift elsewhere. These countries would prefer if others cut their emissions too, thereby avoiding bearing the cost of carbon reduction alone as well as benefiting from matched carbon reduction. To overcome this problem, international coordination in climate policies is required. Vlassis-within a perfectly competitive general equilibrium model of international trade in which pollution is a by-product of production-investigates the welfare properties of a particular form of pollution-tax harmonisation, paying particular attention to the allocation of the revenues collected. The pollution-tax harmonisation reform takes the form of moving the pollution-tax vectors (starting from any initial equilibrium) towards an appropriately weighted vector (with the weights being the countries' output responses to carbon taxes). With respect to the allocation of revenues, the analysis considers two cases: one in which the revenues are distributed to the consumers in a lump sum fashion, and one in which they finance public abatement.

The author shows that, starting from any arbitrary non-symmetric pollution-tax distorted equilibrium, the pollution-tax harmonisation reform delivers potential Pareto improvements. The intuition for this is that the convergence of pollution tax vectors towards a weighted average induces efficiency gains in the production of the tradable goods (and so the intensity of the pollution in all countries) and, as a consequence, tax revenues are being distributed to the consumers making everybody better off. Intuition suggests (and the analytics in the paper confirm) that these efficiency gains remain if public abatement is introduced (financed by the tax revenues), and welfare improvements can be realized under certain conditions on the marginal valuation of those revenues.

The paper suggests avenues for future research such as extending the framework of the analysis to search for reforms that deliver strict Pareto improvements (in the sense that all countries strictly gain from such reforms) and to investigate the role, if any, of non-tradable goods.

\section{Asheim, G., Distributional Argument for Supply-Side Climate Policies}

Climate policies have predominantly focused, with some notable exceptions, on demand-side instruments to control greenhouse gas emissions. This paper puts forward a distributional argument for the use of supply-side policies whereby carbon emissions are controlled through depletion quotas or some permanent confiscation of a fraction of the in situ carbon stock. The distributional effects are identified within the Cobb-Douglas version of the DasguptaHeal-Solow-Stiglitz model of capital accumulation and costless resource extraction. It is assumed that atmospheric absorption capacity is a non-renewable and exhaustible resource, the resource stock is homogenous, and announced policies are perceived to be credible. The author analyses the properties of six policy instruments (implemented by a global government): two demand-side (emission taxes and emission quotas), and four supply-side ones (depletion taxes and depletion quotas which can be auctioned off or allocated freely).

The analytics show that both of the demand-side instruments imply confiscation of the entire resource wealth and the allocation of the property rights of the cumulative absorption capacity emitted resource to the government. If emission quotas are allocated freely, the entire resource wealth is still to be confiscated but the property rights to the cumulative absorption capacity emitted resource input is allocated to the capital owners. Under depletion taxes or auctioned depletion quotas, the results are identical to the corresponding demand policy: 
confiscation of the entire resource wealth and the property rights of the cumulative absorption capacity emitted resource input is allocated to the government. If the depletion quotas are allocated freely, then the property rights are to be allocated to the resource owners which, in turn, implies that net confiscation of the resource stock is limited to the fraction not produced. In a next step, the author examines the possibility of a compensated confiscation of resource stock (meaning that the fraction confiscated is purchased at market prices from the resource owners and put into a permanent reserve). The result suggests that in addition to the fact that the property rights are allocated to the resource owners, they also receive compensation for part of the resource stock: in doing so, this scheme over-compensates the resource owners at the expense of the government, as their income share is increased in absolute terms. Under uncompensated confiscation of the resource stock, the distribution of income between capital owners and resource owners is preserved.

The author also relaxes the assumption of a global government and considers a twocountry world (under competitive markets with perfect capital mobility and free trade in the resource) in which one of the countries is the capital stock and the other the resource stock owner and identifies the distributional effects of the above six policies. The results suggest that only three of the supply-side policies lead to unchanged distribution when moving from a situation where the resource constraint of absorption capacity is binding to a situation where the climate threshold is binding. Moving to partial participation or compliance, demandside policies will be wasteful and have no long-run climate effect. Perhaps not surprisingly, supply-side policies are optimal if implemented by the country endowed with the resource.

The author shows how permanent confiscation, without compensation, of a fraction of the in situ carbon resources, large enough to avoid serious climate change preserves the distribution of income between capital owners and resource owners, compared to the case where no climate policy is in place. Furthermore, he shows that this solution can also be obtained by depletion quotas but not by other considered alternatives. Such observations are of interest as avoiding redistribution may facilitate climate change negotiations.

Finally, the author discusses issues which must be resolved in order to implement a supplyside policy that seeks to leave the distribution unchanged. The first issue is the selection of the medium-extraction-cost carbon resource that should be left in situ and put in a permanent reserve as well as the flexibility of future re-evaluation. The second issue is that owners of medium-extraction-cost carbon resources should be compensated by owners of lowextraction-cost carbon resources. The third issue is that the authority responsible for resource confiscation should be in the resource-host country. Finally, the confiscated resources should not be assumed to be available at a later point, otherwise current fossil fuel prices will not be affected.

\section{Bosetti, V. and E. De Cian, A Good Opening: The Key to Make the Most of Unilateral Climate Action}

Partial cooperation on the provision of a global public good can fail to be environmentally effective. On the one hand, pollution reduction by the coalition might be below the optimal levels of abatement. On the other hand, the reaction of non-signatory countries might be to increase pollution compared to the case when no agreement is in place, typically called leakage. Whether this is the case or not depends on number of forces. In this paper, the authors argue that when a subgroup of countries cooperate on emission reduction, the optimal response of non-signatory countries reflects the interaction between three factors: a) the incentive to free-ride on the environmental benefits of cooperation in the form of reduced 
environmental damages (called damage effect), b) the incentive to expand energy consumption due to a drop in energy prices (referred to as energy market effect) and c) the incentive to adopt cleaner technologies introduced by the coalition (called technology effect). The first and second effects contribute to leakage, the third effect works in the opposite direction. Focusing on partial cooperation between OECD countries (coalition members), the authors' central question is to identify an emission target by the OECD countries that could trigger a positive response by non-signatory nations, i.e. the leakage effect is negative. A central role in answering this question is diffusion of clean technology, including the role of learningby-research and learning-by-doing. Results are tested with a number of robustness checks which include the structure of energy markets, energy supply and international trade elasticities, substitution possibilities in final production, speed of innovation, composition and differences in climate damages.

The authors use the integrated assessment model WITCH (World Induced Technical Change Hybrid model) with endogenous technical change that accounts for the relative price of fossil fuels which depends on the stringency of the climate policy, the scarcity of fossils, the speed of technology diffusion and the price elasticity of energy demand. Furthermore, whether a new technology is adopted by non-signatory nations or not depends also on its price relative to that of fossil fuels. The model incorporates a detailed representation of the energy sector in an inter-temporal growth model of the economy. World's regions make inter-temporally optimal decisions about R\&D-investments, abatement and consumption. The solution of the game is a Nash equilibrium between coalition members acting as a single player, internalising the externality among their members and the remaining non-members, acting as singletons. Fossil fuel prices are influenced by the global use of exhaustible resources and the way technical change is modelled accounts for spillovers of international knowledge and experience. The robustness of the results is tested across alternative model specifications and challenged through extensive sensitivity analysis. The authors recognise that the WITCH model does not represent all possible sources of global interactions. More specifically, international trade of capital and fossil fuels is not fully captured and therefore terms-of trade effects are not considered. Nevertheless, it has to be pointed out that WITCH is probably one of the most detailed integrated assessment models, in particular when considering that the authors analyse not only the Nash equilibrium and the social optimum but also consider the possibility of partial cooperation.

The game-theoretic model comprises a two-stage non-cooperative simultaneous, open membership game. In the first stage, countries decide on their membership and coalitions are formed. In the second stage, countries choose their economic strategies in an inter-temporal setting. In this paper, the authors focus exclusively on the second stage of the game and fix the first stage by focusing on the coalition of OECD countries, abstracting from the analysis whether this coalition would be stable.

Results show that the equilibrium abatement of the coalition composed by OECDcountries would be moderate, in line with pledges subscribed in the Copenhagen Accord. These pledges lead to a reduction of 2050 emission by 30-35 \% below 2005 levels. The mitigation strategy would consist of investments in R\&D in the energy sector and deployment of cleaner technologies with high learning potential. Clearly, this reduction seems small when compared to what would be required to stabilise global warming below $2^{\circ} \mathrm{C}$. However, it is at least a modest step forward and it induces a response by non-OECD countries, which also reduce their emissions even though they are not committed to the climate agreement. This positive response is due to international knowledge spillovers and technology transfers that counterbalance the energy market and damage effect and give non-OECD countries an incentive to switch to cleaner technologies. In other words, international knowledge and tech- 
nology externalities would facilitate the diffusion of cleaner technologies to non-signatory countries, offsetting the free-riding incentive. Technology externalities can occur through climate policy linkages such as the Clean Development Mechanism under the Kyoto Protocol, trade flows and multinational enterprises or skilled-labour mobility.

This result is the consequence of two factors. First, the OECD regions' effort is moderate. A large fraction of damages occurs in non-OECD countries and therefore is not internalised by the OECD coalition. Thus, the damage effect is rather small. Second, as abatement is moderate, the influence on international fuel prices is contained. That is, the energy market effect is relatively small. Both effects would be much higher in a scenario where the OECD group cut emissions beyond the equilibrium level, pursuing reduction rates of 40-45\% below 2005 levels in 2050. It is shown that the benefits of technology externalities in this case would no longer compensate the effect of lower fossil fuel prices and lower damages.

These conclusions suggest that a moderate unilateral climate policy may be more effective than a very ambitious policy and that the international transfers of technologies and knowledge can play an important role for an effective mitigation climate policy. It is clear that such an interesting policy relevant analysis should be extended in future research by explicitly considering stability of coalitions. This would provide a more comprehensive view of which climate policy strategies are effective in a second-best world.

\section{Dekker, T., R. Dellink and J. Ketterer, The Fatter the Tail, the Fatter the Climate Agreement}

International climate negotiations take place in a setting where uncertainties regarding the impacts of climate change are very large. Regional differences in the costs and benefits from emission reductions also complicate the international coordination for combating climate change. The efficient level of mitigation efforts can be attained through global cooperation, e.g. by forming an international environmental agreement (IEA). However, there is a large incentive to free-ride as non-cooperating countries benefit from cooperating countries mitigation efforts. Furthermore, the absence of supra-national authority which can enforce participation and high mitigation levels means that international agreements must be self-enforcing.

One key uncertainty is the probability of very large climate damages, the right-hand side tail of the damage cost distribution. The higher the likelihood of these events, the more they will influence the optimal policy response. In other words, policy response outcomes are highly sensitive to high-consequence low-probability impacts. In this paper, the authors examine the influence of increasing the probability and impact of large climate change damages, also known as the "fat tail" of the damage cost distribution, on the formation, stability and performance of IEAs. Using the stochastic version of the calibrated game-theoretic climate simulation model STACO, the authors systematically vary the shape and location of the distribution of climate change damages to analyse the extent to which IEAs would be affected. The STACO model consists of twelve world regions. Like the model by Bosetti and De Cian, coalition formation is modeled as a two stage process in which regions choose membership in an IEA first and then their economic strategies. Different from this paper, stability of coalition in the first stage is tested by applying the concept of internal and external stability. Due to uncertainty, the concept of stability likelihood is used which has been introduced in the literature before. The model links uncertainties about costs and benefits of mitigation efforts to the stability likelihood of coalitions, relating this to the variance in regional benefit shares of mitigation as well as the variance and level of the global benefit 
parameter. Distributions are generated via Monte Carlo simulations. Asymmetries across coalition members are accounted for through an optimal transfer scheme.

This paper shifts focus from a general uncertainty analysis to assessing the impact of the probability of highly disruptive climate change damages on the stability and performance of climate coalitions. Fat tails in the distribution of mitigation benefits are introduced by increasing the variance of the global benefit parameter and opting for asymmetric distributional shapes. The authors assess the impact of fatter upper tails on stability and the overall performance of an IEA in welfare terms by measuring welfare in relative terms compared to the two benchmarks no and full cooperation. Regions' responses to uncertainty depend on the extent they learn about the true parameter values. Three types of learning are distinguished: no learning, partial learning and full learning.

The simulations confirm that non-participants in an IEA will also undertake abatement efforts relative to their BAU scenario, which increases with expected benefits from mitigation and the fatness of the right-hand side tail of the benefit distribution. Results also show that by not being part of a coalition, regions are likely to conduct less abatement not only because they behave non-cooperatively but also because they do not benefit from burden sharing schemes (monetary transfers) between members of the coalition. Furthermore, the fat-tail scenarios show that countries are willing to form coalitions to reduce the risk of irreversible climate damages. The authors find that both higher expected benefits and fatter upper tails of climate impacts increase the stability likelihood of an international climate agreement and overall welfare. Finally, the authors point out that nevertheless, there remain significant difficulties in striking an ambitious international agreement with broad participation. The payoff functions from this analysis show that mitigation costs are substantial and hence the free-riding incentives make it hard to form large stable coalitions.

In conclusion, increasing the likelihood of very high damages induce that more ambitious coalitions are stable with better performance. In other words, they conclude fatter tails imply more successful, or "fatter" international climate agreements.

\section{References}

IPCC (2007) Climate change 2007. Synthesis Report

Stern N (2006) Stern review: the economics of climate change. Report prepared for the HM Treasury in the UK 